EPJ Web of Conferences 19, 07005 (2012)

DOI: $10.1051 /$ epjconf/20121907005

(C) Owned by the authors, published by EDP Sciences, 2012

\title{
The orbit of the old, metal-rich, open cluster NGC 6791
}

\author{
L. Jílková ${ }^{1,2, a}$, G. Carraro ${ }^{1,3}$, B. Jungwiert ${ }^{4,5}$ and I. Minchev 6 \\ ${ }^{1}$ European Southern Observatory, Santiago, Chile \\ ${ }^{2}$ Dept. of Theoretical Physics and Astrophysics, Faculty of Science, Masaryk University, \\ Brno, Czech Republic \\ ${ }^{3}$ Dipartimento di Astronomia, Universita di Padova, Padova, Italy \\ ${ }^{4}$ Astronomical Institute, Academy of Sciences of the Czech Republic, Prague, \\ Czech Republic \\ ${ }^{5}$ Astronomical Institute, Faculty of Mathematics and Physics, Charles University in Prague, \\ Czech Republic \\ ${ }^{6}$ Astrophysikalisches Institut Potsdam, Potsdam Germany
}

\begin{abstract}
We present a study of the Galactic orbit of the old, metal-rich open cluster NGC 6791. To recover its recent kinematical history we integrate the cluster's orbit backward in a Milky Way (MW) model including bar and spiral structure. To estimate the viability of one of the cluster's proposed scenarios of origin we also follow a set of forward integrations originating closer to the Galactic center (GC) where the metal enrichment could be faster. In presence of bar and spiral arms the orbits experience the radial migration due to resonance overlap mechanism [8] and so could possibly reach the recent orbit of NGC 6791.
\end{abstract}

\section{INTRODUCTION}

NGC 6791 is a unique stellar system among Galactic open clusters - a very old (7-10 Gyr, see [3, 7]) and extremely metal-rich $([\mathrm{Fe} / \mathrm{H}] \sim+0.4$, see $[4])$, located inside the solar circle (at the galocentric radius of $8 \mathrm{kpc}$, around $0.8 \mathrm{kpc}$ above the Galactic plane, see [3]), and possibly experienced prolonged star formation [12]. The combination of these properties is puzzling and the origin of the cluster still remains unclear. One of possible scenarios is that the cluster had formed close to the GC and later has migrated outwards to its current location. Multiple rotating patterns cause a strong radial migration due to their resonance overlap as described by [8], therefore we investigate the cluster's orbit in the MW model including the bar and spiral arms components.

\section{BACKWARD AND FORWARD ORBIT INVESTIGATION}

To compute the orbit we adopt a model of gravitational potential consistent with the MW. The axisymmetric time-independent background is based on model by [1] scaled to the recent values of rotation curve [11], where most of the bulge mass is replaced by the bar. The Galactic bar is modeled as Ferrers potential of inhomogeneous triaxial ellipsoid [10], $\Omega_{\mathrm{bar}}=60 \mathrm{~km} / \mathrm{s} / \mathrm{kpc}$, and other bar parameters taken from [9]. We also include 2 spiral arms modeled as a spiral perturbation by [5], $\Omega_{\mathrm{SA}}=20 \mathrm{~km} / \mathrm{s} / \mathrm{kpc}$

\footnotetext{
ae-mail: 1jilkova@eso.org
}

This is an Open Access article distributed under the terms of the Creative Commons Attribution-Noncommercial License 3.0, which permits unrestricted use, distribution, and reproduction in any noncommercial medium, provided the original work is properly cited. 

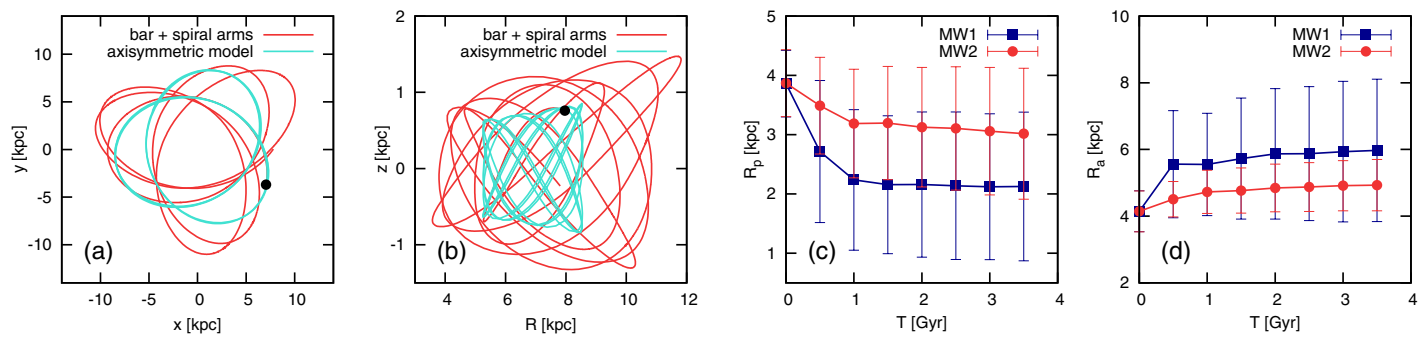

Figure 1. (a), (b): recent orbit of NGC 6791-projection of the orbit into the Galactic plane (a), and into the meridional plane (b). Orbits in the pure axisymmetric potential and in the model including bar and spiral arms are shown with blue and red lines, respectively. Integration was backwards for time of $1 \mathrm{Gyr}$. (c), (d): time evolution of average values of orbital parameters for the present-day MW model MW1, and for the migration tuned model MW2. Both models include the bar and spiral arms non-axisymmetric components. Error-bars have sizes of standard deviations of distributions of the orbital parameters at given time for the whole set of orbits, but note that the distributions might strongly deviate from Gaussian.

To investigate the recent orbit of NGC 6791 we carry on backward integrations for $1 \mathrm{Gyr}$, starting from the cluster's current position and velocity vectors obtained from the most recent observational data found in literature - celestial coordinates, distance to the Sun [3], radial velocity, and proper motions both from [2], see Figs. 1 (a) and (b) for the orbit. The observational uncertainties of the data are taken into account in Monte Carlo fashion by integrating a set of 1000 orbits.

To estimate the probability and efficiency of the radial migration mechanism due to the resonance overlap [8] we also follow a set of $10^{4}$ initially-close-to-circular orbits distributed between 3 and $5 \mathrm{kpc}$ from the GC and we integrate them forward for time of $4 \mathrm{Gyr}$. The MW model as described above is scaled to reproduce the present-day observations of the MW, we denoted this model as MW1. Except for the initial growth of the rotating patterns, the model is steady-state. Therefore we also consider a second version of the model-denoted as MW2 - with parameters more supporting the resonance overlap migration process. MW2 has a more massive bar, stronger spiral arms, and angular velocities are changed to bring resonances closer.

Figs. 1 (c) and (d) show the outcome of this experiment-time evolution of apo-galacticons and peri-galacticons of integrated orbits. As expected, the MW2 model can produce more eccentric orbits with higher apo-galacticons and smaller peri-galacticons. We found that during the $4 \mathrm{Gyr}$ integration time, the MW2 model can produce orbits with apo- and peri-galacticons close to the actual values for NGC 6791. A more detailed description of the work presented here can be found in [6].

LJ acknowledges the support by grants No. 205/08/H005 (Czech Science Foundation) and MUNI/A/0968/2009 (Masaryk University in Brno). BJ was supported by the grant LC06014-Center for Theoretical Astrophysics (Czech Ministry of Education) and by the research plan AV0Z10030501 (Academy of Sciences of the Czech Republic).

\section{References}

[1] Allen, C. and Santillan, A., Revista Mexicana de Astronomia y Astrofisica 22, (1991) 255-263

[2] Bedin, L.R. et al., A\&A 460, (2006) L27-L30

[3] Brogaard, K. et al., A\&A 525, (2011) A2+

[4] Carraro, G. et al., ApJ 643, (2006) 1151-1159

[5] Cox, D.P. and Gómez, G.C, APJ 142, (2002) 261-267 
Assembling the Puzzle of the Milky Way

[6] Jílková, L., Carraro, G., Jungwiert, B., and Minchev, I., submitted to A\&A (2011)

[7] King, I.R. et al., AJ 130, (2005) 626-634

[8] Minchev, I. and Famaey, B., ApJ 722, (2010) 112-121

[9] Pichardo, B., Martos, M. and Moreno, E., ApJ 609, (2004) 144-165

[10] Pfenniger, D., A\&A 134, (1984) 373-386

[11] Reid, M.J. et al., ApJ 700, (2009) 137-148

[12] Twarog, B.A., Carraro, G. and Anthony-Twarog, B.J., ApJL 727, (2011) L7+ 